\title{
A SIMPLE CONSTRUCTION FOR RIGID AND WEAKLY HOMOGENEOUS BOOLEAN ALGEBRAS ANSWERING A QUESTION OF RUBIN ${ }^{1.2}$
}

\author{
GARY BRENNER
}

\begin{abstract}
We introduce a method for constructing Boolean algebras from trees which preserves some of the trees properties. The method is used to produce a very simple construction for rigid Boolean algebras and to construct a weakly homogeneous Boolean algebra without homogeneous factors.
\end{abstract}

0. Introduction. In this paper we present two constructions: a weakly homogeneous Boolean algebra that answers a question of Rubin, and what we feel is the simplest construction of a rigid Boolean algebra.

A rigid Boolean algebra is one which has no nonidentity automorphism. We begin our construction of a rigid Boolean algebra by taking a tree $T$ satisfying (a) the height of $T$ is $\omega$; (b) $T$ has a single root; (c) for all distinct $\alpha, \beta \in T$, the number of immediate successors to $\alpha$ is different from the number of immediate successors to $\beta$; and (d) the number of immediate successors is always regular. We form "wedges", $S_{\alpha}=\{\beta \in T: \alpha \leqslant \beta\}$, for each $\alpha \in T$, and close the set of wedges under finite unions and complements. The result is our algebra.

Many constructions of rigid Boolean algebras have been given. In [VDMR] there is a short history. van Douwen, Monk and Rubin ask for a "natural" construction of a rigid Boolean algebra. We offer our construction as a candidate.

van Douwen, in [vD] has a similar construction. He begins with a tree $T$ satisfying (a) and (c) and with the property that for all $\alpha \in T, \kappa_{\alpha}$, the number of immediate successors to $\alpha$, satisfies $\kappa_{\alpha}^{\kappa_{0}}=\kappa_{\alpha}$. He then topologizes the tree by taking certain infinite unions of wedges. The result is a rigid 0-dimensional compact space. The algebra of closed-and-open sets is the desired rigid Boolean algebra.

A Boolean algebra $B$ is homogeneous if for any nonzero $b \in B$, the set $\{a \in B$ : $a \leqslant b$ \} viewed as a Boolean algebra is isomorphic to $B$. It is weakly homogeneous if for all distinct nonzero $a, b \in B$ there exist nonzero $b_{1} \leqslant b, a_{1} \leqslant a$ such that the algebras consisting of $\left\{c: c \leqslant b_{1}\right\}$ and $\left\{c: c \leqslant a_{1}\right\}$.are isomorphic. We answer a question of Rubin in $[\mathbf{R}]$ by constructing a weakly homogeneous Boolean algebra that has no homogeneous factors.

Received by the editors January 27, 1982 and, in revised form, October 25, 1982.

1980 Mathematics Subject Classification. Primary 06E99.

'These results are contained in Chapter 2 of the author's $\mathrm{Ph}$. D. dissertation, prepared under the direction of J. D. Monk at the University of Colorado. The author expresses his gratitude to Professor Monk.

${ }^{2}$ This paper is dedicated to Merry Havens. 
In a subsequent paper we will present more general results concerning Boolean algebras obtained from trees in the fashion described here. We call this class of Boolean algebras tree algebras. See [B1 and B2] for more results involving tree algebras.

We wish to thank J. D. Monk for many useful comments and suggestions in writing this paper.

\section{Basic definitions and facts.}

Definition 1.1. (a) $\langle T, \leqslant\rangle$ is a tree iff $\leqslant$ partially orders $T$ and $\forall \alpha \in T,\{\beta \in T$ : $\beta \leqslant \alpha\}$ is well ordered by $\leqslant$. We will use $T$ to denote both the set of nodes and the tree. $\alpha$ is a root of $T$ iff $\alpha$ is minimal in $T$. For all $\alpha \in T$ we let $A_{\alpha}$ denote the set of $\leqslant$-immediate successors to $\alpha$. Two elements $\alpha, \beta$ are comparable iff $\alpha \leqslant \beta$ or $\beta \leqslant \alpha$. Otherwise they are incomparable. For all $\alpha \in T$, level $\alpha=$ order type $\{\beta$ : $\beta<\alpha\}$. We define ht $T=\sup \{($ level $\alpha)+1: \alpha \in T\}$.

The symbol $\leqslant$ will also be used to denote order in Boolean algebras. Thus for $a, b$ in Boolean algebra $B, a \leqslant b$ iff $a \cdot-b=0$. The exact meaning of $\leqslant$ will be clear from context. For any set $L$ we let card $L$ denote the cardinality of $L$.

(b) For any tree $T$ we define $B_{T}$, the tree algebra generated from $T$, as follows. For all $\alpha \in T$, let $S_{\alpha}=\{\beta \in T: \alpha \leqslant \beta\}$; then define $B_{T}$ as the closure of $\left\{S_{\alpha}: \alpha \in T\right\}$ under finite unions and complements relative to the set $T$.

$B_{T}$ is a Boolean algebra under the operations of set union, intersection and complement. In this paper when we use the word "algebra" we mean "Boolean algebra." We now give some easy technical results.

Definition 1.2. (a) Given algebra $B$ and $b \in B, B \uparrow b$ denotes the algebra whose universe is $\left\{a \in B: a \leqslant{ }_{B} b\right\}$ with operations ${ }^{+} B\left|b,{ }^{\circ} B\right| b$ restrictions of the corre-

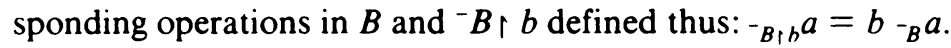

(b) Given tree $T$ and $\alpha \in T, T, \alpha$ denotes the tree whose universe is $\{\beta \in T$ : $\alpha \leqslant \beta$ \} ordered by restricting the ordering on $T$.

LEMMA 1.3. Let $T$ be a tree.

(a) $\forall b \in B_{T} \backslash\{0\}, b=\sum_{i \in n} f_{i}$ with $n$ finite and $f_{i} \cdot f_{j}=0$ for $i \neq j$ and each $f_{i}$ of the form $f_{i}=S_{\alpha_{i}}-\sum_{\beta \in J_{1}} S_{\beta}\left(J_{i}\right.$ a finite set of successors to $\left.\alpha_{i}\right)$ unless $T$ has more than one root in which case $f_{i}$ may also have the form $f_{i}=-\sum_{\beta \in J} S_{\beta}$ (J finite).

(b) $\left\{S_{\alpha}: \alpha \in T\right\} \cup\left\{b \in B_{T}: b\right.$ is an atom $\}$ is dense in $B_{T}$.

(c) If $\beta \in T$ then $B_{T} \mid S_{\beta}=B_{T \uparrow \beta}$.

Proof. (a) $\left\{S_{\alpha}: \alpha \in T\right\}$ generates $B_{T}$ so $b=\sum_{i \in n} \prod_{j \in J,} S_{\alpha_{t, j}}^{\varepsilon_{t, j}}$ where $\varepsilon \in\{-1,1\} ; S_{\alpha}^{-1}$ $=-S_{\alpha} ; S_{\alpha}^{l}=S_{\alpha} ; n, J_{1}$ finite; and for $i \neq i^{\prime}$,

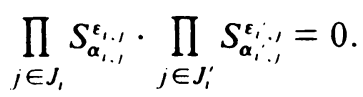

Each

$$
\prod_{j \in J_{i}} S_{\boldsymbol{\alpha}_{\imath, j}}^{\boldsymbol{\varepsilon}_{i, j}}=\prod_{j \in J_{i}^{\prime}} S_{\boldsymbol{\alpha}_{\imath, j}} \cdot \prod_{j \in J_{i,}^{\prime \prime}}-S_{\boldsymbol{\alpha}_{t, .}}
$$


and $\forall i \in n$ either $\prod_{j \in J_{i}^{\prime}} S_{\alpha_{t, 1}}=S_{\alpha_{t, 1}}$ for some $j \in J_{i}^{\prime}$ or $\prod_{j \in J_{i}} S_{\alpha_{t, 1}}=0$. The desired result now follows when we note that if $\alpha$ is the unique root of $T$ and $J$ is finite then $-\Sigma_{\beta \in J} S_{\beta}=S_{\alpha}-\Sigma_{\beta \in J} S_{\beta}$.

(b) The result follows immediately from (a) when we note that $b$ is an atom iff for some $\alpha \in T, A_{\alpha}$ is finite and $b=S_{\alpha}-\Sigma_{\beta \in A_{\alpha}} S_{\beta}$.

(c) This is clear.

\section{The construction.}

Definition 2.1. Let $B$ be an algebra and $b \in B$. We say that $P \subset B$ is a partition of $B($ or of $b$ ) iff

1. $\sum P=1_{B}($ or $b)$,

2. for all distinct $p, q \in P, p \cdot q=0$ and

3. $0 \notin P$.

LEMMA 2.2. Let $\kappa$ be an uncountable regular cardinal and $T$ a tree satisfying

1. ht $T \leqslant \omega$,

2. $\forall \alpha \in T$, card $A_{\alpha} \neq \kappa$,

3. $T$ has a single root

then $B_{T}$ does not have a partition of cardinality $\kappa$.

Proof. Suppose that $T$ is a tree satisfying the hypotheses and $P=\left\{e_{1}: i \in \kappa\right\}$ is a partition of $B_{T}$ with $e_{i} \neq e_{j}$ for $i \neq j$. Without loss of generality we may assume that each $e_{i}$ is of the form $e_{i}=S_{\alpha_{i}}-\sum_{j \in J_{i}} S_{\beta_{i,}}$ with $J_{i}$ finite and $\alpha_{i}<\beta_{i, j}$ for all $i, j$. Let $H_{P}=\left\{\alpha_{i}: i \in \kappa\right\}$; then card $H_{P}=\kappa$ and $\alpha_{i} \neq \alpha_{j}$ for $i \neq j$ since $e_{i} \cdot e_{j}=0$ for $i \neq j$. $T$ has at most $\omega$ levels and $\mathrm{cf} \kappa>\omega$ so $\kappa$-many elements of $H_{P}$ occur on the same level.

Let $r$ be the least element of $\omega$ such that there is a tree $T$ satisfying

1. ht $T \leqslant \omega$,

2. $\forall \alpha \in T$ card $A_{\alpha} \neq \kappa$,

3. $T$ has a single root,

4. $B_{T}$ has a partition $P$, each element of the form $S_{\alpha}-\Sigma_{\beta \in J} S_{\beta}$ with

$J$ finite and $\alpha<\beta$ for all $\beta \in J$; and card $P=\kappa$,

5. $H_{P}$ has $\kappa$-many elements on level $r$.

Since the trees that we are considering each have a single root, $r \neq 0$. Let $T, P, H=H_{P}$ witness our choice of $r$. Let $\alpha$ be the root of $T$. We will derive a contradiction.

Case 1. card $A_{\alpha}>\kappa$. We claim that $\alpha \in H$.

For suppose $\alpha \notin H$ then $\forall \delta \in H, S_{\beta}$ lies beneath exactly one element of $\left\{S_{\gamma}\right.$ : $\left.\gamma \in A_{\alpha}\right\}$, say $S_{\gamma_{\delta}} \cdot \forall \delta \in H, S_{\delta} \leqslant S_{\gamma_{\delta}}$ so $\cup_{\delta \in H} S_{\delta} \subseteq \cup_{\delta \in H^{\prime}} S_{\gamma_{\delta}}$. But $\sum_{\delta \in H^{\prime}} S_{\delta}=1$ so $\sum_{\delta \in H} S_{\gamma_{\delta}}=1$. This is possible only if $\left\{\gamma_{\delta}: \delta \in H\right\}=A_{\alpha}$. Thus card $H \geqslant \operatorname{card} A_{\alpha}>\kappa$, a contradiction. So $\alpha \in H$.

So we have that for some $i \in \kappa$ and finite $J, e_{i}=S_{\alpha}-\Sigma_{\beta \in J} S_{\beta}$. Note that $\forall \beta \in J, e_{j} \cdot S_{\beta} \neq 0 \Rightarrow e_{j} \leqslant S_{\beta}$ hence $\forall \beta \in J, S_{\beta}=\Sigma\left\{e_{i}: e_{i} \leqslant S_{\beta}\right\}$ so $\forall \beta \in J,\left\{e_{i}: e_{i} \leqslant\right.$ $\left.S_{\beta}\right\}$ is a partition of $S_{\beta}$. Since $J$ is finite, we can pick $\beta \in J$ so that

$$
\operatorname{card}\left\{e_{i}: \alpha_{i} \text { is on level } r \text { and } e_{i} \leqslant S_{\beta}\right\}=\kappa .
$$


Now $\left\{e_{1}: e_{1} \leqslant S_{\beta}\right\}$ is a partition of $B \wedge S_{\beta}$, so by Lemma 1.3 (c) $P^{*}=\left\{e_{1}: e_{1} \leqslant S_{\beta}\right\}$ is a partition of $B_{7\} \beta}$ and $T, \beta, P^{*}, H_{P^{*}}$ contradict the choice of $r$.

Case 2. card $A_{\alpha}<\kappa$. Then for some $\beta \in A_{\alpha}$. card $\{\gamma \in H: \gamma$ is on level $r$ and $\beta<\gamma\}=\kappa$. The argument used in Case 1 shows that $T, \beta, P^{*}, H_{p^{*}}$ again contradict the choice of $r$.

Derinition 2.3. (a) $B$ is a rigid algebra iff the identity is the only automorphism of $B$.

(b) $B$ is a homogeneous algebra iff $\forall$ nonzero $b \in B, B \mid b \cong B$. B is a neakly homogeneous algebra iff $\forall$ nonzero $a, b \in B, \exists$ nonzero $a_{1} \leqslant a, b_{1} \leqslant b$ such that $B \nmid a_{1} \cong B \nmid b_{1}$.

THEOREM 2.4. Let $T$ be a tree satisfying

1. ht $T=\omega$ and $T$ has a single root,

2. $\forall \alpha, \beta \in T\left(\alpha \neq \beta \Rightarrow \operatorname{card} A_{\alpha} \neq \operatorname{card} B_{\beta}\right)$.

3. $\forall \alpha \in T$. card $A_{\alpha}$ is regular and infinite.

Then $B_{T}$ is a rigid algebra.

Proof. Claim 1. $\forall \alpha \in T, \forall$ uncountable, regular $\kappa, S_{\alpha}$ has a partition of size $\kappa$ iff $\exists \beta \geqslant \alpha$, card $A_{\beta}=\kappa$. The implication from right to left is trivial. To go from left to right, suppose there is no $\beta \geqslant \alpha$ with card $A_{\beta}=\kappa$. Then $\kappa, T \uparrow \alpha$ satisfy the hypotheses of Lemma 2.2 so $B_{T_{\uparrow} \alpha}$ has no partition of size $\kappa$. Thus $B \uparrow S_{\alpha}$ has no partition of size $\kappa$.

Now suppose that $\varphi$ is an automorphism of $B$.

Claim 2. $\forall \alpha \in T, \varphi\left(S_{\alpha}\right) \leqslant S_{\alpha}$. If the claim is false then there is some $b \in B_{T}$ such that $0 \neq b \leqslant \varphi\left(S_{\alpha}\right) \cdot-S_{\alpha}$. Since $\forall \alpha \in T . A_{\alpha}$ is infinite; by the remark in the proof of Lemma 1.3(b), $B_{T}$ contains no atoms. So by 1.3(b), there is some $\gamma \in T$ such that $S_{\gamma} \leqslant b$. Thus $\varphi\left(S_{\alpha}\right)$ has a partition of size card $A_{\gamma}$. Since $S_{\gamma} \neq S_{\alpha \gamma}, \alpha \neq \gamma$, so by the first claim $S_{\alpha}$ has no partition of size card $A_{\gamma}$. This contradicts the fact that $\varphi$ is an isomorphism and we have Claim 2.

Suppose $\exists \alpha \in T . \varphi\left(S_{\alpha}\right) \neq S_{\alpha}$. Then $\exists b \in B_{T}, 0 \neq b \leqslant S_{\alpha} \cdot-\varphi\left(S_{\alpha}\right)$. We pick $\gamma \in T$ such that $S_{\gamma} \leqslant b$. Then $\varphi\left(S_{\gamma}\right) \leqslant \varphi\left(S_{\alpha}\right)$. But $S_{\gamma} \leqslant-\varphi\left(S_{\alpha}\right)$ so $\varphi\left(S_{\gamma}\right) \leqslant S_{\gamma} \leqslant-\varphi\left(S_{\alpha}\right)$. This implies that $\varphi\left(S_{\gamma}\right)=0$, a contradiction. This completes the proof.

THEOREM 2.5. There exists a weakly homogeneous tree algebra with no homogeneous factors.

Proof. Let $T$ be the tree of ht $\omega$ with one root satisfying $\forall \alpha \in T$ (level $\alpha=n \Rightarrow$ card $A_{\alpha}=\omega_{n}$ ).

$B=B_{T}$ has no homogeneous factors: Suppose $B \cong C \times D$ then $C \cong B \nmid b$ for some $b \in B$. Pick $\alpha, \beta \in T$ such that $S_{\alpha}, S_{\beta} \leqslant b$ and level $\alpha<$ level $\beta$. Let $n=$ level $\alpha$. By Lemma 2.2, $B \uparrow S_{\alpha}$ has a partition of size $\omega_{n}$ but $B \uparrow S_{\beta}$ does not. Thus $B \mid b$ is not homogeneous.

$B_{T}$ is weakly homogeneous: Take any nonzero $a_{1}, b_{1} \in B_{T}$. Find $\alpha, \beta \in T$ such that $S_{\beta} \leqslant a_{1}, S_{\beta} \leqslant b_{1}$ and level $\alpha=$ level $\beta$. So $B \backslash S_{\alpha} \cong B \uparrow S_{\beta}$.

M. Rubin asked in $[\mathbf{R}]$ whether a weakly homogeneous algebra without homogeneous factors exists and if such an algebra can be complete. Solovay $[\mathbf{S}]$ and, 
independently, Koppelberg in $[\mathbf{K}]$ showed that no such complete algebra exists. (Our construction suggests the following question.

Question. For all $\kappa$ is there a $\kappa^{+}$-complete weakly homogeneous algebra with no homogeneous factors? In particular, suppose for cardinal $\kappa, T$ is a tree of ht $\omega$ satisfying $\forall \alpha \in T$ card $A_{\alpha}$ is regular and greater than $\kappa$ and $\forall \alpha, \beta \in T(\alpha \neq \beta$, card $A_{\alpha} \neq$ card $\left.A_{\beta}\right)$. Let $B_{T}^{\kappa}$ be the closure of $\left\{S_{\alpha}: \alpha \in T\right\}$ under $\kappa$-unions and complements. Is $B_{T}^{\kappa}$ a weakly homogeneous algebra without homogeneous factors?

ADDED IN PROOF. (1) J. D. Monk solved the question we ask by obtaining a canonical form for $B_{T}^{\kappa}$. The following observation of S. Koppelberg, which is probably known, replaces a more complicated lemma of Monk.

Lemma A. Let $T$ be a set, $X$ a collection of subsets of $T, \kappa$ any infinite cardinal and $A$ the closure of $X$ under complements relative to $T$ and under unions of $\leqslant \kappa$ elements. Then for each $a \in A$, there is $Y \in[X]^{\leqslant \kappa}$ and $F \subset Y_{2}$ such that $a=\cup_{f, F} \cap_{1, r^{*}}$.

Lemma A immediately yields:

LEMMA B. If $\kappa$ is an infinite cardinal and $T$ is a single rooted tree of height $\leqslant \omega$, then every nonzero $b \in B_{T}$ can be written in the form $b=\sum_{1-\lambda} f_{1}$, with (a) $\lambda \leqslant 2^{\kappa}$. (b) $f_{1} \cdot f_{t}=0$ for $i \neq j$ and $f_{t} \neq 0$ for each $i$, and (c) $f_{i}=S_{t}-\sum_{u \in J_{i}} S_{u}$ for some $J_{1}$, a set of pairwise incomparable successors to $t_{i}$ of power $\leqslant \kappa$.

Now using proofs almost identical to those used to show Lemma 2.2 and Theorems 2.4 and 2.5 Monk obtains, respectively, Lemma C and Theorems I and II.

LEMMA C. Let $\kappa$ and $\mu$ be regular cardinals, $\kappa \geqslant \omega$ and $\mu \geqslant 2^{\kappa}$ and $T$ be a tree satisfving (a) ht $T \leqslant \omega$. (b) $\forall t \in T$, card $A_{t} \neq \mu$, and (c) $T$ has a single root, then $B_{T}$ does not have a partition of cardinality $\mu$.

THEOREM I. If $\kappa$ is a regular infinite cardinal and $T$ satisfies

(a) ht $T=\omega$ and $T$ has a single root,

(b) $\forall s, t \in T, s \neq t \Rightarrow$ card $A_{s} \neq$ card $A_{t}$.

(c) $\forall s, t \in T$, card $A_{t}$ is regular and card $A_{t} \geqslant 2^{\kappa}$, then $B_{T}$ is rigid.

THEOREM II. If $T$ is a single rooted tree of height $\omega$ such that $\forall t \in T$. level $t=n \rightarrow$ card $A_{t}=\left(2^{\kappa}\right)^{+(n+1)}$, then $B_{T}^{\kappa}$ is weakly homogeneous and has no homogeneous factor.

(2) Subsequent to Monk, S. Todorčević constructed $\kappa$-complete weakly homogeneous BA's with no homogeneous factors in cardinalities lower than Monk's. For all regular $\boldsymbol{\aleph}_{0} \leqslant \kappa \leqslant \lambda$, Todorčević constructs $2^{\lambda}$ nonisomorphic $\kappa$-complete BA's of cardinality $\lambda^{\kappa}$. So, for instance, he has a $\sigma$-complete example of power $2^{\kappa^{\prime}}$. Monk's $\sigma$-complete algebras have power $\geqslant\left(\left(2^{\aleph_{0}}\right)^{+\omega}\right)^{\aleph_{0}} \geqslant\left(2^{\aleph_{0}}\right)^{+\omega}$.

\section{REFERENCES}

[B1] G. Brenner, A new rigid Boolean algebra, Abstracts Amer. Math. Soc. 1 (1980). 389.

[B2] Tree algebras. Abstracts Amer. Math. Soc. 2 (1981), 270-271.

[vD] E. K. van Douwen, An easy compact zero-dimensional rigid space.

[vDMR] E. K. van Douwen, J. D. Monk and M. Rubin. Some questions about Boolean algebras, Algebra Universalis 11 (1980), 220-243. 
[K] S. Koppelberg, A lattice structure on the isomorphism types of complete Boolean algehras, Set Theory and Model Theory, Proceedings, Bonn 1979, vol, 872. Springer-Verlag, Berlin and New York, 1981, pp. 98-126.

[R] M. Rubin, On the reconstruction of Boolean algebras from their automorphism groups, Arch. Math. Logik Grundlag. 20 (1980), 125-146.

[S] R. M. Solovay (unpublished).

Department of Mathematics, University of COlorado, Boulder, COlorado 80309

Current address: Department of Mathematics, University of Hawaii, Honolulu, Hawaii 96822 96822 